\title{
Hilfestellung in der Hausaufgabenbetreuung und den Lernzeiten aus der Perspektive von Schüler*innen
}

\author{
Markus Sauerwein • Pia Rother
}

Eingegangen: 21. April 2021 / Überarbeitet: 1. September 2021 / Angenommen: 12. Oktober 2021 / Online publiziert: 23. Februar 2022

(C) Der/die Autor(en) 2022

Zusammenfassung Im Beitrag wird anhand von Daten der Studien StEG-S und StEG-Tandem mit einem Mixed Method-Ansatz untersucht, wie Schüler*innen Hilfestellung in der Hausaufgabenbetreuung und Lernzeiten wahrnehmen. Die Forschung zu Hausaufgaben und individuellem Unterricht zeigt, dass Lehrkräfte vor der Herausforderung stehen, Schüler*innen zeitgleich Hilfe zukommen zu lassen, was potenziell eher für leistungsschwächere Schüler*innen ohne familiale Unterstützung relevant wäre. Empirisch zeigen sich jedoch keine Teilnahmeunterschiede an der Hausaufgabenbetreuung nach Leistungen und sozialer Herkunft, wie mithilfe von Daten der beiden Studien analysiert wird. Mittels Latenter Profilanalysen werden drei Profile der wahrgenommenen Hilfe der Schüler*innen auf Basis StEG-Tandem Daten dargestellt - wenig, mittlere und viel Hilfe. Diese Profile unterscheiden sich nicht in ihrer Zusammensetzung hinsichtlich Leistung und sozialer Differenzkategorien. Ferner zeigt die Auswertung der neun Gruppendiskussionen der StEG-Tandem Daten mit je drei bis acht Schüler*innen, dass das Warten auf Hilfe eher kein Problem darstellt und sie bei der Aufgabenbearbeitung nicht zwingend Hilfe benötigen bzw. nicht von allen ,echte' Hilfe erhalten.

Schlüsselwörter Ganztagsschule · Hausaufgaben · Lernzeit · Individuelle Förderung · Mixed Method

Prof. Dr. Markus Sauerwein

Fliedner Fachhochschule Düsseldorf, Geschwister-Aufricht-Straße 9, 40489 Düsseldorf, Deutschland

DIPF I Leibniz-Institut für Bildungsforschung und Bildungsinformation, Frankfurt a. M., Deutschland E-Mail: Sauerwein@dipf.de; Sauerwein@ fliedner-fachhochschule.de

Vertr.-Prof.in Dr. Pia Rother

Institut für Sozialwesen, Universität Kassel, Arnold-Bode-Str. 10, 34127 Kassel, Deutschland

E-Mail: prother@uni-mainz.de

Johannes Gutenberg-Universität Mainz, Mainz, Deutschland 


\title{
Help in homework supervision and study periods from the perspective of students
}

\begin{abstract}
In this paper, we use data from the StEG-S and StEG-Tandem studies with a mixed method approach to examine how students perceive assistance in homework supervision and study periods in schools. Research on homework supervision and individualized education shows that teachers face the challenge of providing support to many students simultaneously (Breidenstein et al. 2017), which may be more relevant for lower-performing students without familial assistance. Empirically, however, there are no differences in participation in homework supervision according to performance and social background, as analyzed using data from the two studies. Using latent profile analyses, three profiles of students' perception of help are presented based on StEG-Tandem data-little, medium, and much help. These profiles do not differ in their composition in terms of performance and social difference categories. Furthermore, the analysis of the nine group discussions of the StEG-Tandem with three to eight students each show that waiting for help is not an issue for students and that they do not necessarily need help when working on tasks or rather not every staff provides help perceived as useful to the students.
\end{abstract}

Keywords All-day-school - Supervised homework · Study periods · Individual support $\cdot$ Mixed method

\section{Einleitung}

Die Erledigung von Hausaufgaben erfolgt durch den kontinuierlichen Ausbau von ganztägigen Bildungs- und Betreuungsarrangements zunehmend in institutionellen Settings. Hausaufgaben werden somit zu Schulaufgaben und von Kindern institutionalisiert in Hausaufgabenbetreuungen oder Lernzeiten bearbeitet (Grimm und Schulz-Gade 2017). Für diesen Wandel können zwei zentrale Argumente angeführt werden: Erstens erfüllen Hausaufgaben-Settings eine kustodiale Funktion, wenn Kinder mehr Zeit in der Schule verbringen, damit ihre Eltern anderen Beschäftigungen nachgehen können. Zweitens werden neben dem Betreuungsargument die erzieherische und didaktische Funktion von Hausaufgaben (Kohler 2017, S. $18 \mathrm{ff}$.) angeführt, dass Schüler*innen, insbesondere aus ressourcenärmeren Familien, bei der Aufgabenbearbeitung in der Hausaufgabenbetreuung Hilfestellungen erhalten. So sollen auch soziale Disparitäten ausgeglichen werden (Nordt 2020).

Der vorliegende Beitrag geht zunächst der Frage nach, ob leistungsschwächere Schüler*innen und/oder Schüler*innen aus eher sozial benachteiligten Familien die Hausaufgabenbetreuung häufiger in Anspruch nehmen oder eher das Betreuungsargument ausschlaggebend ist. Zweitens untersuchen wir mittels Latenter Profilanalysen, ob unterschiedliche Profile von Schüler*innen in der wahrgenommenen Hilfe durch die Betreuer*innen existieren und wie sich diese Profile in den sozioökonomischen Hintergrundvariablen sowie hinsichtlich der Schulleistung unterscheiden. Hierfür kategorisieren wir die Schüler*innen zunächst, um drittens objektiv-herme- 
neutisch über die Analyse von Gruppendiskussionen einen tiefergehenden Einblick auf Hilfestellung aus der Perspektive von Schüler*innen zu erhalten.

\section{Forschungsstand}

Mit ganztagsschulischen Konzepten wird u. a. das Ziel verfolgt, Schüler*innen mehr individuelle Förderung zukommen zu lassen und dadurch auch herkunftsbedingte Disparitäten auszugleichen und zugleich Familien - de facto meist Müttern - eine (umfangreichere) Berufstätigkeit zu ermöglichen und hinsichtlich der Vereinbarkeit von Familie und Beruf zu entlasten (u. a. Züchner und Fischer 2014). Entsprechend dieser konzeptionellen Zielsetzung wurden in den vergangenen Jahren zahlreiche Studien durchgeführt, die untersuchen, inwiefern Ganztagsschulen bzw. bestimmte Ganztagsangebote zu einer Leistungssteigerung beitragen und kompensierend wirken (Holtappels et al. 2019; Steinmann und Strietholt 2019; Strietholt et al. 2015), aber auch inwiefern bestimmte Förderangebote auch stigmatisierend sein können (Sauerwein und Heer 2020). Zusammenfassend zeigen diese Studien jedoch, dass Ganztagsschule nicht wie erhofft zu einer Verbesserung schulischer Leistungen beiträgt (Sauerwein et al. 2019; Sauerwein und Rother im Druck).

\subsection{Hausaufgabenbetreuung in der Ganztagsschule}

Obwohl sich die Schulleistungen durch die Ganztagsteilnahme eher nicht verbessern, wird der betreuten Bearbeitung von Hausaufgaben - die in Ganztagsschulen angeboten werden und mit einer Reorganisation der Hausaufgaben einhergehen - ein positives Potenzial zugeschrieben, Benachteiligungen abzubauen und die Schulleistungen zu verbessern. Argumentiert wird, dass Kinder im Vergleich zur häuslichen Hausaufgabenerledigung hier mehr individuelle Unterstützung bei der Aufgabenbearbeitung erhalten könnten und so auch Bildungsungleichheiten reduziert werden (Brisson und Theis 2020; Dohrmann et al. 2021; Gaiser und Kielblock 2019; Nordt 2020).

De facto sind Hausaufgabenbetreuungen bzw. Lernzeiten die häufigsten Ganztagsangebote und werden nahezu an jeder Ganztagsschule angeboten (Gaiser et al. 2020; StEG-Konsortium 2019). Dies ist möglicherweise darauf zurückzuführen, dass Eltern bei der Anmeldung zum Ganztag der Hausaufgabenbetreuung eine hohe Bedeutung beimessen (Beher et al. 2007; Nordt und Röhner 2008). Sie wünschen sich durch die Hausaufgabenbetreuung gezielte Hilfestellung für ihr Kind (Altermann et al. 2016), wobei diese Erwartung jedoch meistens nicht erfüllt wird (Killus und Tillmann 2017). Ebenfalls wird von Eltern das sogenannte „Restpensum“ beklagt, welches Schüler*innen trotz Hausaufgabenbetreuung zu erledigen haben (Gängler und Markert 2011). Insgesamt machen die Hausaufgaben inzwischen (in der Zeit vor Covid) einen sinkenden Anteil im Alltag von Familien aus (BMFSFJ 2021), was auch als Hinweis auf eine Verlagerung der Hausaufgaben in den schulischen Kontext gelesen werden darf. 


\subsection{Lernzeiten und Hausaufgabenbetreuung}

In Ganztagsschulen finden sich zwei Formate, in denen Schüler*innen Aufgaben in der Schule bearbeiten sollen: Lernzeiten und Hausaufgabenbetreuung (Maschke et al. 2019). Während in der Hausaufgabenbetreuung nur ein Teil der Schüler*innen (freiwillig) an bestimmten Wochentagen teilnimmt (und somit auch in unterschiedlichen Gruppenkonstellationen), finden Lernzeiten zumeist im Klassenverband statt und sind entsprechend verpflichtend. Ebenso wird angenommen, dass Lernzeiten eher von Lehrkräften durchgeführt werden, während in der Hausaufgabenbetreuung (auch) anderes pädagogisch tätiges Personal anwesend ist (Brisson et al. 2017, 2019; Maschke et al. 2019). Belastbare (repräsentative) Ergebnisse liegen hierzu jedoch nicht vor. In Studien wird selten zwischen beiden Formaten differenziert, in der Praxis ist die Trennung zwischen beiden Formaten fließend und an Schulen bestehen auch Doppelstrukturen, d. h. es werden sowohl Hausaufgabenbetreuung als auch Lernzeiten angeboten. Tendenziell ist ein vorsichtiger Trend zu erkennen, die Hausaufgabenbetreuungen in Lernzeiten umzuwandeln (Gaiser et al. 2020).

\subsection{Individualisierung des Unterrichts}

Ebenso wie die Institutionalisierung von Hausaufgaben kann individualisierter Unterricht als Reaktion auf unterschiedliche Anforderungen des Bildungssystems verstanden werden. Beispiele hierfür sind gleichfalls der Umgang mit sozialen Ungleichheiten, die Umsetzung der Inklusion von Schüler*innen mit Förderbedarfen sowie Bestrebungen zum längeren gemeinsamen Lernen (Dumont 2019; Klieme und Warwas 2011). Im individualisierten Unterricht wird auf die „Synchronisation der Schülertätigkeiten“ (Breidenstein und Rademacher 2017, S. 1) verzichtet ,zugunsten einer Diversifizierung und eben ,Individualisierung ““ (Breidenstein und Rademacher 2017, S. 1). Individualisierter Unterricht soll sowohl einer Überforderung der , langsameren" als auch der Langeweile der ,schnelleren' Schüler*innen entgegenwirken und somit zu einer Effizienzsteigerung der Unterrichtszeit führen (Breidenstein und Rademacher 2013; Breidenstein et al. 2017). Deutlich wird auch eine Verlagerung der Verantwortung des Nutzens der Unterrichtszeit: Während (vor allem in quantitativen Studien) die Verantwortung des guten Unterrichts der Lehrkraft obliegt und Teil des Konzeptes von Unterrichtsqualität ist (Klieme und Rakoczy 2008; Klieme 2019; Praetorius et al. 2020), zeigen qualitative Studien (u. a. Breidenstein und Rademacher 2013; Breidenstein et al. 2017), dass es zu einer Verschiebung der Verantwortung für die Nutzung der Unterrichtszeit von der Lehrperson zu den Schüler*innen kommt. Die Geschwindigkeit, in der Schüler*innen in dem vorgegebenen Zeitraum ihre Aufgaben erledigen, wird so zu einem Differenzierungskriterium, das ,sich vereinfacht auf die Formel bringen lässt: Je schneller desto besser" (Budde 2012, S. 180). Die schnelleren Schüler*innen werden durch Gratifikationen belohnt - etwa dem Malen, welches jedoch nur einen (leisen) Beschäftigungszweck erfüllt - oder erhalten Zusatzaufgaben, während die Langsameren keine Gratifikationen erhalten, aber auch keine zusätzlichen Bildungsinhalte und somit Lerngelegenheiten. So bleiben auch in einem individualisierten Unterricht gemeinsame Fixpunkte bestehen, wie etwa 
ein „kollektiver Anfangs- und Endpunkt der Arbeitszeit“ (Breidenstein et al. 2017, S. 50).

Für Lehrkräfte bringt individualisierter Unterricht neue Herausforderungen mit sich, da die Schüler*innen nicht mehr an gleichen, sondern an unterschiedlichen Aufgaben in ihrem jeweiligen Lerntempo arbeiten und somit Lehrkräfte auf unterschiedliche Individualanliegen der Schüler*innen zeitgleich reagieren sollen. So bilden sich beispielsweise Warteschlangen, Lehrkräfte eilen von Schulkind zu Schulkind, Schüler*innen ,klammern' eine Wäscheklammer mit ihrem Namen an ein Band $^{1}$, das die Reihenfolge der Warteschlange öffentlich festhält und zugleich als „Disziplinierungspraktik“ fungiert (Wolter 2017). Schüler*innen übernehmen aber auch untereinander die Funktion als Helfende (Breidenstein et al. 2017).

\subsection{Betreute Hausaufgaben - ein Format der individuellen Förderung?}

Elemente individuellen Unterrichts und Lernens können auch in der betreuten Bearbeitung von Hausaufgaben enthalten sein, wenn Schüler*innen eigenständig, in ihrem Lerntempo an teilweise differenten Aufgaben arbeiten. Sie sind ebenso mit dem Versprechen verknüpft, individuelle Förderung zu ermöglichen und Schüler*innen zu unterstützen, die im außerinstitutionellen Bereich keine adäquate Hilfe erhalten, sei es, weil sie allein zu Hause sind oder die Eltern die notwendige Unterstützung nicht leisten wollen/können (Nordt und Röhner 2008; Rabenstein und Podubrin 2015). Vorliegende Arbeiten berichten, dass die Hausaufgabenbetreuung (in der Sekundarstufe I) eher von Schüler*innen aus sozioökonomisch benachteiligten Haushalten bzw. mit Migrationshintergrund besucht wird (Steiner 2009), wobei insgesamt die Teilnahme an Ganztagsschulen (im Primarbereich) eher geringen Selektionseffekten unterliegt zu Gunsten soziökonomisch besser gestellter Kinder (Marcus et al. 2016). Empirisch beobachtet werden konnte ein stärkeres Gefühl der Unterstützung in der Hausaufgabenbetreuung bei Schüler*innen mit Migrationshintergrund (Tillack et al. 2015). Weitere Arbeiten liegen hierzu nicht vor.

Hilfestellung bildet dementsprechend eine zentrale Dimension dafür, dass Schüler*innen von der Hausaufgabenbetreuung oder den Lernzeiten profitieren können. Kritisch diskutiert wird jedoch, dass es dem Personal in der Hausaufgabenbetreuung oftmals nicht gelingt, Kinder hinreichend fachlich zu unterstützen - auch aufgrund der Gruppengröße in der Hausaufgabenbetreuung (Rother 2019). Ebenso fühlen sich Lehrkräfte jenseits ihres Faches zumeist nicht zuständig, um Schüler*innen bei der Aufgabenbearbeitung zu unterstützen und kennen die individuellen Lernvoraussetzungen und -ziele der Schüler*innen nicht (Rabenstein und Podubrin 2015). Zudem scheinen Lehrkräfte einen intensiven Austausch über einzelne Schüler*innen kaum als nötig zu empfinden (Chiapparini 2019).

Im Fokus steht zumeist die Quantität der zu erledigenden Hausaufgaben, weniger die Qualität (Rabenstein und Podubrin 2015) sowie die Umsetzung von Ordnungsstrukturen und Umgangsregeln (Hess 2020; Nordt und Röhner 2008). Rabenstein und Podubrin (2015) können in der Hausaufgabenbetreuung zwar kurze Situationen individueller Zuwendung beobachten, in ,denen pädagogische Praktiken des

\footnotetext{
1 Diese Praktik des Klammerns berichteten in der Studie 2 sowohl Schüler*innen als auch Lehrkräfte.
} 
Kontrollierens und korrigierenden Eingreifens“ (Rabenstein und Podubrin 2015, S. 237) dominieren. Das Kontrollieren bezieht sich zumeist auf die Vollständigkeit der Aufgaben, für das sich die Betreuenden verantwortlich sehen, jedoch bleibt eine gezielte Unterstützung des Verstehens aus, weil die Betreuenden permanent für alle ansprechbar sein müssen (Rabenstein und Podubrin 2015). So steht oftmals die Arbeitsfähigkeit einzelner Schüler*innen im Fokus, wobei kaum Unterschiede zwischen Lernzeiten und Hausaufgabenbetreuung bestehen (Rabenstein und Podubrin 2015), die sich in struktureller Hinsicht vor allem dadurch unterscheiden, dass die Lernzeit im Klassenverband stattfindet und die Hausaufgabenbetreuung davon abgekoppelt ist. Demgegenüber zeigen jedoch Brisson und Theis (2020), dass die Qualität von Lernzeitaufgaben höher eingeschätzt wird als die in der Hausaufgabenbetreuung und sich Schüler*innen bei der Bearbeitung der Aufgaben in Lernzeiten auch als kompetenter erleben. Dies führt jedoch nicht zu Leistungszuwächsen (Brisson und Theis 2020). Auch berichten Schüler*innen, dass es ihnen oft schwer fällt, sich bei hoher Lautstärke in der Hausaufgabenbetreuung oder den Lernzeiten zu konzentrieren (Brisson et al. 2019). Gaiser und Kielblock (2019) fassen entsprechend zusammen, „dass Hausaufgaben an Ganztagsschulen vielfach noch als klassische Hausaufgaben konzeptioniert sind [...] und dass es [...] um das Erledigen der Hausaufgaben geht und nicht um das Lernen und die Beschäftigung mit Lerninhalten“ (Gaiser und Kielblock 2019, S. 164).

Ebenso kann argumentiert werden, dass im Kontrast zum individualisierten Unterricht - der einen neuen oder anderen Umgang mit Zeit unter dem Motto ,alle in ihrem eigenen Tempo“ erfordert (Breidenstein und Rademacher 2013; Budde 2012) - diese Argumentation brüchig ist. Bei der Aufgabenbearbeitung zu Hause besteht tatsächlich die Chance, im eigenen Tempo zu arbeiten, während innerhalb der Aufgabenerledigung in der Schule klare Anfangs- und Endzeitpunkte bereits gesetzt sind. So bleibt letztlich - neben der Betreuungsfunktion - nur die bessere Hilfestellung für Schüler*innen durch Lehrkräfte oder Personal, die jedoch - wie aufgezeigt - eher unzureichend ist.

\section{Fragestellung}

Entsprechend des Forschungsstandes, ist offen, inwiefern leistungsschwächere Schüler*innen sowie Schüler*innen aus eher bildungsbenachteiligten Haushalten besonders von der Hausaufgabenbetreuung profitieren könnten. Neben dem Betreuungsargument wird die potenzielle Hilfestellung und individuelle Unterstützung der Lehrkräfte bzw. des Personals als Begründung für die Erledigung von Aufgaben in der Schule aufgeführt. Zwar liegen Arbeiten vor, die die Hilfegewährung durch das Personal untersuchen (Rabenstein und Podubrin 2015) oder allgemein die Situation in der betreuten Aufgabenbearbeitung beschreiben (Nordt und Röhner 2008), aber Studien, die die von den Schüler*innen erlebte Hilfestellung betrachten, stellen ein Forschungsdesiderat dar. Hierbei gehen wir davon aus, dass Schüler*innen die Hilfestellung unterschiedlich wahrnehmen. Entsprechend sollen in dem Beitrag folgende Fragestellungen beantwortet werden: 
1. Inwiefern nehmen Schüler*innen die Hausaufgabenbetreuung in Anspruch, die schlechtere Schulleistungen aufweisen und/oder zu Hause weniger Unterstützung von ihren Eltern erwarten können?

2. Bestehen unterschiedliche Profile von Schüler*innen in der wahrgenommenen Hilfe durch die Betreuer*innen? Unterscheiden sich diese Profile in den sozioökonomischen Hintergrundvariablen sowie der Schulleistung?

3. Wie wird die Hilfe durch Lehrkräfte oder Betreuer*innen seitens der Schüler*innen als Unterstützung wahrgenommen? Wie erleben die Schüler*innen die Hilfe bei der Aufgabenerledigung?

\section{Methoden}

Um die Frage zu beantworten, welche Kinder die Hausaufgabenbetreuung aufsuchen, greifen wir die Studien StEG-S und StEG-Tandem zurück, die beide Schüler*innen in der fünften Jahrgangsstufe befragen. Während in StEG-S zu dem hier betrachteten zweiten Messzeitpunkt (Schulhalbjahr) insgesamt 1969 Schüler*innen aus 66 Schulen unterschiedlicher Schulformen (5,6\% Hauptschule I 6,8\% Realschule | $30,8 \%$ Schulen mit mehreren Bildungsgängen I 26,6\% Gesamtschule | 30,3\% Gymnasium) an der Studie teilnahmen (Sauerwein 2017), ist in StEG-Tandem die Stichprobe für den zweiten Messzeitpunkt zum Schulhalbjahr mit 212 Schüler*innen aus fünf Gesamtschulen in Hessen deutlich geringer und umfasst nur Schüler*innen an Integrierten Gesamtschulen (Brisson et al. 2021). ${ }^{2}$ In StEG-Tandem wurden jedoch die Hilfe in den Hausaufgaben via Fragebogen im Detail erhoben und darüber hinaus mit einigen der Schüler*innen Gruppendiskussionen durchgeführt. StEG-S liefert entsprechend aufgrund der größeren Stichprobe eine validere Datengrundlage und umfasst mehrere Schulformen. StEG-Tandem ermöglicht aufgrund der zusätzlichen qualitativen Daten tiefergehende Einblicke, so dass wir auf beide Studien zurückgreifen. ${ }^{3}$ Hinsichtlich der Ganztagsform finden sich in beiden Studien sowohl Ganztagsschulen mit freiwilligen und verbindlichen Teilnahmemodi (StEG-S: 20 Schulen mit verpflichtenden Teilnahmemodi I StEG-Tandem: drei Schulen mit freiwilliger Hausaufgabenbetreuung, zwei Schulen mit verpflichtenden Lernzeiten). In StEG-S wird zudem nicht zwischen Lernzeiten und der Hausaufgabenbetreuung differenziert, während StEG-Tandem theoretisch eine Unterscheidung zulässt, jedoch die Stichprobengröße dann zu klein wird, so dass wir hierauf verzichten mussten.

\footnotetext{
2 Bei StEG-Tandem handelt es sich zudem um eine Interventionsstudie. Wir greifen nur auf die Daten der Kontrollgruppe zurück, die keine Intervention erfahren hat.

3 Die diesem Beitrag zugrundeliegenden Forschungsprojekte StEG-S und StEG-Tandem wurden aus Mitteln des Bundesministeriums für Bildung und Forschung (BMBF) gefördert. Die Verantwortung für den Inhalt dieser Veröffentlichung liegt bei den Autor*innen. Die hier präsentierten Sekundäranalysen wurden in 2020 gefördert durch das Zentrum für Schul-, Bildungs- und Hochschulforschung an der Johannes Gutenberg-Universität Mainz.
} 


\subsection{Quantitative Methoden zur Erfassung der Schülerperspektive}

In den beiden Studien kamen unterschiedliche Leistungstests zum Einsatz. In StEG-S wurde die Lesegeschwindigkeit über das Salzburger Lese-Screening (SLS) erhoben. Hierbei wurden den Schüler*innen insgesamt 70 Sätze vorgelegt und sie mussten innerhalb von drei Minuten entscheiden, ob die jeweiligen Sätze inhaltlich richtig sind (Auer et al. 2005). Das Leseverstehen wurde über den Frankfurter Leseverständnistest (FLVT) erfasst (Souvignier et al. 2008) und über die gesamte Stichprobe hinweg skaliert (für eine ausführliche Darstellung siehe Sauerwein 2017). Die verwendeten Daten beziehen sich auf den zweiten Messzeitpunkt zum Schulhalbjahr. Die Schüler*innen haben auch angegeben, ob sie an der Hausaufgabenbetreuung teilgenommen haben und machten Angaben zu ihren Zeugnisnoten in Deutsch und Mathematik. Daneben liegen mit dem HISEI (highest international socio-economic index of occupational status) und Angaben zu den Büchern im Haushalt ( $1=0-10$ Bücher bis $5=$ über 200 Bücher) Informationen zur sozialen Herkunft der Schüler*innen vor. Ebenso wurde erhoben, ob die Schüler*innen einen Migrationshintergrund haben (selbst oder mindestens ein Elternteil im Ausland geboren) und ein Gymnasium besuchen.

In StEG-Tandem wurden die Kompetenzen der Schüler*innen in Englisch und Mathematik über den Quop-Test (Souvignier et al. 2014) erfasst, ebenso wie die Noten in diesen beiden Fächern (Brisson et al. 2021). Zudem liegen Angaben über die Bücher im Haushalt (1=0-10 Bücher bis 5=über 200 Bücher) und den Migrationshintergrund der Schüler*innen vor. Darüber hinaus geben die Daten von StEGTandem noch tiefergehende Einblicke, über die Hilfe in der Hausaufgabenbetreuung (HAB) bzw. den Lernzeiten (LZ). Dazu wurden die Schüler*innen gefragt: Was denkst du über die Hilfe der Betreuer*innen in der HAB/LZ? Meine Betreuer*innen/ Lehrer*innen ... und konnten dort nachfolgende Items (Tab. 1) auf einer vierstufigen Skala $(1=$ stimmt gar nicht | $2=$ stimmt eher nicht | 3=stimmt eher | $4=$ stimmt genau) beantworten (Chronbachs Alpha 0,77 । MW: 3,09। SD: 0,57).

Um die erste Fragestellung zu beantworten, wurde basierend auf den Daten von beiden Studien mittels t-test berechnet, ob sich an Hausaufgabenbetreuung bzw. Lernzeiten teilnehmende Schüler*innen hinsichtlich der sozialen Herkunft und/oder der Schulleistungen von den nicht teilnehmenden Schüler*innen unterscheiden.

Tab. 1 Hilfe in der Hausaufgabenbetreuung/den Lernzeiten - StEG-Tandem

\begin{tabular}{llll}
\hline & MW & SD & $N$ \\
\hline ... wissen genau, was ich gut kann & 2,88 & 0,95 & 118 \\
... wissen genau, was ich nicht so gut kann & 2,71 & 1,06 & 119 \\
... geben mir Hinweise, wie ich eine Aufgabe gut lösen kann & 3,29 & 0,91 & 118 \\
... lassen mir Zeit für meine Antworten & 3,09 & 0,89 & 118 \\
... sagen mir die Lösung vor & 1,58 & 0,82 & 118 \\
... loben mich, wenn ich eine schwere Aufgabe geschafft habe & 2,82 & 1,06 & 117 \\
... helfen mir dabei, Fehler in meinen Aufgaben zu entdecken & 3,13 & 0,87 & 117 \\
... können gut erklären, wenn ich eine Frage habe & 3,33 & 0,86 & 119 \\
\hline
\end{tabular}


Für die Frage nach den „Hilfeprofilen“, also der durch die Schüler*innen wahrgenommenen Unterstützung, haben wir Latente Profilanalysen (LPA) durchgeführt. In LPAs werden Objekte (hier Schüler*innen) anhand ihrer Merkmalsprofile in möglichst wenige Gruppen zusammengefasst, die nach innen homogen und nach außen möglichst heterogen sind (Giegler und Rost 1993). Dabei steht ein Set von Antwortmustern für ein bestimmtes Profil, wobei die Anzahl der zu bestimmenden Profile zunächst unbekannt ist (Magidson und Vermunt 2004; Gollwitzer 2012). Jede*r Schüler*in wird so mit einer bestimmten Wahrscheinlichkeit einem Profil zugeordnet (Kaplan und Keller 2011).

Für die Bestimmung der Anzahl der Profile können unterschiedliche Indikatoren herangezogen werden. Bei dem Akaike Informations Criterion (AIC), dem Bayesian Information Critierion (BIC) und dem adjustierten Bayesian Information Critierion (aBIC), zeigen kleinere Werte an, dass die angenommene Anzahl an Profilen gut auf die Daten passt. Der Lo-Mendel-Rubin-Test (LMR-Test) und der Bootstrapped Liklihood Ratio Test (BLR-Test) prüft, ob die angenommene Profillösung signifikant besser zu den Daten passt, als eine Lösung mit einem Profil weniger (Nylund et al. 2007). Letztlich müssen die Profile aber auch sinnvoll interpretierbar sein. D.h. es gilt Lösungen zu vermeiden, bei denen Profile entstehen, die anteilmäßig nur wenige - in unserem Fall - Schüler*innen repräsentieren (Magidson und Vermunt 2004; Kaplan und Keller 2011). Gerade der LMRT und der BLRT sind zeitaufwendige Verfahren, weshalb Morin et al. (2016) ein zeitsparendes und praktikables Vorgehen vorschlagen: Demnach sollen Fit-Indikatoren grafisch abgebildet werden und wenn es zu keiner bedeutsamen Verbesserung der Fit-Werte kommt (es einen „Knick“ gibt), wird die Profillösung akzeptiert, ab der es zu keiner bedeutsamen Verbesserung des Fits kommt. Für die so ermittelten Lösungen haben wir zusätzlich den LMRTest und BLR-Test durchgeführt.

\subsection{Qualitative Methoden zur Erfassung der Schülerperspektive}

Um tiefgehender die Perspektiven von Schüler*innen auf Hausaufgaben in Ganztagsschulen zu untersuchen, greifen wir zusätzlich auf die Gruppendiskussionen in StEG-Tandem zurück, die mit den Schüler*innen zu Beginn des zweiten Schulhalbjahres geführt wurden (Brisson et al. 2021). Insgesamt wurden zu dem entsprechenden Zeitpunkt neun Gruppendiskussionen geführt, deren Teilnehmer*innenZahl zwischen drei und acht Schüler*innen lag. Zwei dieser Gruppendiskussionen eine in einer Schule mit Lernzeiten, eine in einer Schule mit Hausaufgabenbetreuung -, die basierend auf den Ergebnissen der Latenten Profilanalysen ausgewählt wurden und auf der manifesten Ebene das Thema der Hilfestellung behandeln, wurden mit der Objektiven Hermeneutik (Wernet 2009) ausgewertet. In der Objektiven Hermeneutik wird davon ausgegangen wird, dass gesellschaftliche Strukturen Handlungen erzeugen, die nicht intentional sind, und dass das soziale Geschehen (un)bewusst davon geleitet wird, wie die soziale Wirklichkeit interpretiert wird, ohne bereits ontologisch vorgegeben zu sein. Das Ziel der Methode ist es demnach, die latente Sinnstruktur der zu untersuchenden Welt - in dieser Sekundäranalyse der Hausaufgabenbetreuungen und der Lernzeiten aus der Perspektive von Schüler*innen (statt Pädagog*innen) - ,verstehend zu erfassen“ (Wernet 2009, S. 11, Herv. i. O.). Die 
Objektive Hermeneutik ermöglicht also die Rekonstruktion der latenten Sinnstruktur des Textes - in dieser Analyse der Gruppendiskussionstranskripte - entlang geltender Regeln. Die Regeln bestimmen auch die Handlungsoptionen, jedoch welche realisiert werden, entscheidet sich in der jeweiligen Fallstruktur (Wernet 2009, S. 15). Es geht also darum, objektiv-hermeneutisch zu rekonstruieren, welche ,Regelgeleitetheit sozialen Handelns" (Wernet 2009., S. 13, Herv. i. O.) sich zeigt. In den Gruppendiskussionen positionieren sich die Schüler*innen im Verhältnis zu ihren Mit-Schüler*innen zu bestimmten Themen und dadurch werden die Perspektiven der Schüler*innen innerhalb der Gruppe beispielsweise zum Thema Hilfestellung rekonstruierbar. Das Qualitätskriterium der Datentiefe zu einem bestimmten latenten Sinngehalt, wie zur Hilfestellung, wird hier über die Erhebung in der Gruppe erreicht.

Die zwei Gruppendiskussionen, die basierend auf den Ergebnissen der Latenten Profilanalysen sowie hinsichtlich ihres zunächst manifesten Sinngehalts kontrastiv ausgewählt wurden, konnten anhand von je vier Sequenzen vollständig rekonstruiert werden. Eine Darstellung der gesamten Rekonstruktion ist nicht möglich, weshalb im Folgenden exemplarisch je eine markante Sequenz dargestellt wird.

\section{Ergebnisse}

Nachfolgend werden die Ergebnisse entlang der drei Fragestellungen dargestellt.

\subsection{Wer nimmt an der Hausaufgabenbetreuung teil?}

Um zu betrachten, inwiefern sich Schüler*innen, die eine Hausaufgabenbetreuung besuchen, von denen unterscheiden, die dies nicht tun, haben wir auf die Daten der Studien StEG-S und StEG-Tandem zurückgegriffen und mittels t-test analysiert, ob sich die Schüler*innen in den Schulleistungen und der sozialen Herkunft unterscheiden.

\subsubsection{Unterschiede zwischen Schüler*innen in der Leistung}

Die StEG-S Daten zeigen, dass 60,4\% der Schüler*innen in der fünften Klasse, an der Hausaufgabenbetreuung (oder freiwilligen Lernzeit) teilnehmen. Es zeigen sich allerdings keine signifikanten Unterschiede in der Leseleistung und dem Lesever-

Tab. 2 Unterschiede in der Leistung der Schulkinder - StEG-S

\begin{tabular}{llllr}
\hline & $\begin{array}{l}\text { Keine Teilnahme } \\
\text { MW (SD) }\end{array}$ & $\begin{array}{l}\text { Teilnahme an HAB/LZ } \\
\text { MW (SD) }\end{array}$ & $p$ & $N$ \\
\hline Leseverstehen (FLVT) & $0,02(1,17)$ & $-0,01(1,14)$ & 0,63 & 1958 \\
$\begin{array}{l}\text { Lesegeschwindigkeit } \\
\text { (SLS) }\end{array}$ & $42,58(10,56)$ & $42,52(10,52)$ & 0,91 & 1962 \\
Note Deutsch Halbjahr & $2,69(0,90)$ & & & \\
Note Mathe Halbjahr & $2,65(0,99)$ & $2,65(0,89)$ & 0,37 & 1743 \\
\hline
\end{tabular}


Tab. 3 Unterschiede in der Leistung der Schulkinder - StEG-Tandem

\begin{tabular}{lllll}
\hline & $\begin{array}{l}\text { Keine Teilnahme } \\
\text { MW (SD) }\end{array}$ & Teilnahme an HAB/LZ & $p$ & $N$ \\
\hline Englisch Test (Quop) & $0,30(1,15)$ & $0,08(1,34)$ & 0,21 & 205 \\
Mathe Test (Quop) & $0,15(1,31)$ & $-0,16(1,24)$ & 0,09 & 206 \\
Englisch Note Halbjahr & $2,39(0,94)$ & $2,20(1,01)$ & 0,16 & 207 \\
Mathe Note Halbjahr & $2,51(0,84)$ & $2,44(0,94)$ & 0,16 & 207 \\
\hline
\end{tabular}

stehen. Ebenfalls unterscheiden sich die Schulnoten in den Fächern Deutsch und Mathematik nicht (Tab. 2).

Wird die kleinere Stichprobe aus StEG-Tandem herangezogen, bestätigt sich dieses Bild. Ebenfalls bestehen keine Unterschiede in der Englisch- und Mathematikleistung sowie in den Schulnoten zwischen den Teilnehmer*innen und NichtTeilnehmer*innen an der Hausaufgabenbetreuung bzw. den Lernzeiten (Tab. 3).

\subsubsection{Unterschiede zwischen Schüler*innen nach sozialer Herkunft}

Eine weitere Begründungslinie, warum Schüler*innen eine Hausaufgabenbetreuung in der Schule besuchen sollen, zielt auf die ggf. mangelnde Unterstützung durch die Eltern ab. Ergebnisse von StEG-S zeigen, dass hinsichtlich des HISEI und der Bücher im Haushalt keine Unterschiede zwischen Schüler*innen bestehen, die die Hausaufgabenbetreuung oder Lernzeiten nutzen, und denen, die ihre Hausaufgaben außerhalb der Schule erledigen. Schüler*innen mit Migrationshintergrund scheinen tendenziell eher seltener die Hausaufgabenbetreuung aufzusuchen (der Befund ist jedoch nicht signifikant). Auch besuchen Schüler*innen am Gymnasium seltener die Hausaufgabenbetreuung (Tab. 4).

Tab. 4 Unterschiede in der sozialen Herkunft der Schüler*innen und besuchten Schulform - StEG-S

\begin{tabular}{llllr}
\hline & Keine Teilnahme & Teilnahme an HAB/LZ & $p$ & $N$ \\
& MW (SD) & MW (SD) & & 1658 \\
\hline HISEI & $45,50(21,01)$ & $43,95(20,38)$ & 0,14 & 1952 \\
Bücher im Haushalt & $3,11(1,08)$ & $3,17(1,09)$ & 0,23 & 1958 \\
Migrationshintergrund & $42,4 \%$ & $57,6 \%$ & 0.07 & \\
Kein Migrationshinter- & $38,1 \%$ & $61,9 \%$ & & \\
grund & & & & \\
Gymnasium & $52,2 \%$ & $47,8 \%$ & & \\
Kein Gymnasium & $33,9 \%$ & $66,1 \%$ & &
\end{tabular}

Tab. 5 Unterschiede in der sozialen Herkunft der Schulkinder und besuchten Schulform - StEG-Tandem

\begin{tabular}{lllllr}
\hline & $\begin{array}{l}\text { Keine Teilnahme } \\
\text { MW (SD) }\end{array}$ & $\begin{array}{l}\text { Teilnahme an } \\
\text { HAB/LZ } \\
\text { MW (SD) }\end{array}$ & Cramer-V & $p$ & $N$ \\
\hline Bücher im Haushalt & $2,81(1,35)$ & $2,81(1,29)$ & - & 0,974 & 208 \\
Migrationshintergrund & 46,4 & 53,6 & 0,09 & 0.19 & 208 \\
$\begin{array}{l}\text { Kein Migrationshin- } \\
\text { tergrund }\end{array}$ & 36,8 & 63,2 & & \\
\hline
\end{tabular}


In StEG-Tandem wurden zur Erfassung der sozialen Herkunft nur der Migrationshintergrund und der Bücherumfang im Haushalt erhoben. In beiden Variablen zeigen sich keine statistisch bedeutsamen Unterschiede zwischen den Teilnehmenden an der Hausaufgabenbetreuung und den Schüler*innen, die nicht an der Hausaufgabenbetreuung partizipieren (Tab. 5).

Trotz der selektiven Stichprobe (nur fünf Gesamtschulen) liefert StEG-Tandem also keine anderen Ergebnisse als die Daten von StEG-S, was uns empirisch begründet dazu dient, den Fokus auf StEG-Tamdem zu richten. Für die nachfolgenden zwei Fragestellungen greifen wir deshalb - wie beschrieben - nur noch auf die StEGTandem Daten zurück, da zudem entsprechende Variablen in StEG-S nicht erhoben wurden.

\subsection{Hilfe in der Hausaufgabenbetreuung bzw. den Lernzeiten durch Lehrkräfte oder Betreuer*innen}

Betrachtet man lediglich den Mittelwert der Skala der Hilfe durch die Lehrkräfte bzw. Betreuer*innen in der Hausaufgabenbetreuung bzw. den Lernzeiten (MW: 3,09 । SD: 0,57, s. Methoden), so könnte man davon ausgehen, dass an Ganztagsschulen insgesamt die Schüler*innen, die Hilfestellung als positiv bewerten. Für einen differenzierteren Blick haben wir aber Latente Profilanalysen durchgeführt. Ferner haben wir untersucht, inwiefern sich diese Profile in den sozioökonomischen Hintergrundvariablen sowie der Schulleistung unterscheiden.

Hierbei deuten die Fit Indikatoren darauf hin, dass eine drei Profil umfassende Lösung am besten zu den Daten passt (Abb. 1). Der LMRT zeigt, dass eine zwei Profil-Lösung der drei Profil-Lösung überlegen ist, der BLRT deutet jedoch auf das Gegenteil hin. Ähnlich verhält es sich, wenn eine vier Profile umfassende Lösung angenommen wird. Der LMRT sieht eine drei Profil-Lösung als überlegen an, der

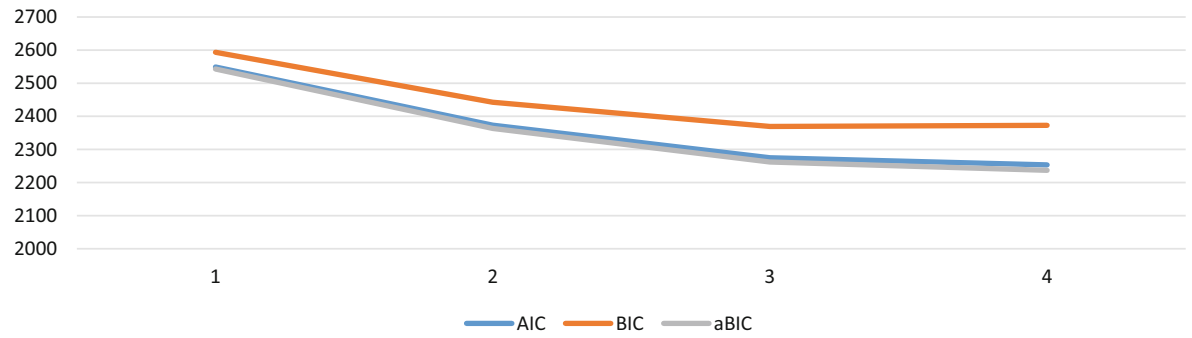

Abb. 1 Ermittlung der Anzahl der Profile x-Achse Anzahl der Klassen

Tab. 6 Übersicht Entscheidung Profilklassen

\begin{tabular}{llllll}
\hline Anzahl Profile & AIC & BIC & aBIC & LMRT (k-1) & BLRT (k-1) \\
\hline 1 & 2549,010 & 2593,476 & 2542,894 & - & - \\
2 & 2373,036 & 2442,514 & 2363,480 & 0,01 & 0,00 \\
3 & 2274,906 & 2369,396 & 2261,909 & 0,14 & 0,00 \\
4 & 2253,339 & 2372,841 & 2236,901 & 0,30 & 0,00 \\
\hline
\end{tabular}




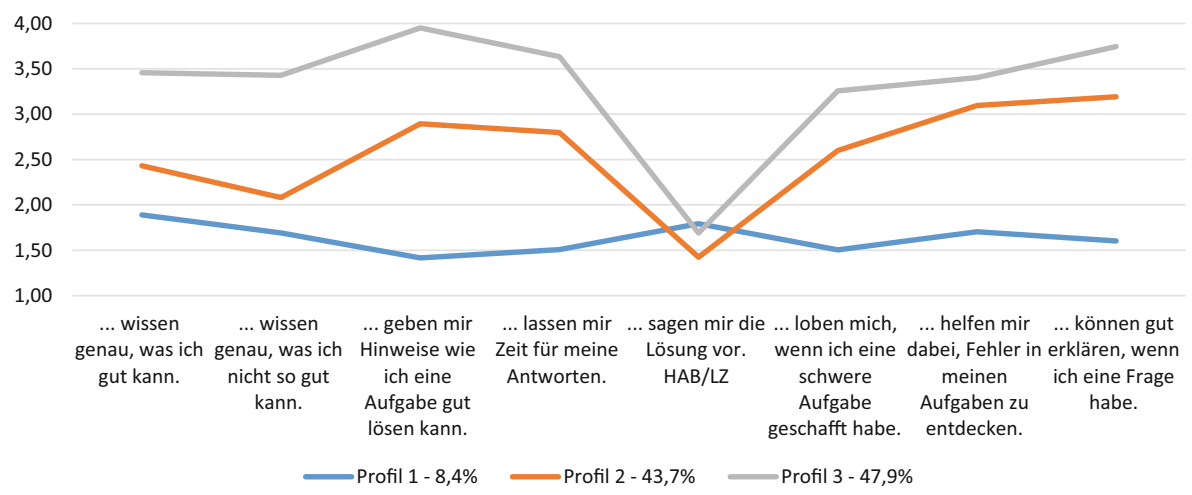

Abb. 2 Hilfe durch Lehrkräfte/Betreuer*innen

BLRT eine vier Profil-Lösung. Letztlich haben wir uns für drei Profile entschieden, da AIC, BIC und aBIC sich noch substantiell verbessern, zwischen einer zwei und drei Profile umfassenden Lösung, jedoch nicht mehr von einer drei zu einer vier Profile umfassenden Lösung (Tab. 6). Im Ergebnis zeigen sich drei ausdifferenzierte Profile, die nachfolgend beschrieben werden.

\subsubsection{Profil 1}

Profil 1 umfasst lediglich 8,4\% ( $N=10$ Schüler*innen). Diese Schüler*innen berichten insgesamt in allen abgefragten Variablen von einer geringen Unterstützung durch Lehrkräfte oder Betreuer*innen. Ebenso erhalten sie wenig Lob (Abb. 2). Die Schüler*innen dieses Profils finden sich je zur Hälfte in Schulen mit Lernzeiten und Hausaufgabenbetreuung. Auch können sie in jeder der fünf Schulen beobachtet werden. Ebenfalls sind die dichotomen Geschlechterkategorien gleich verteilt. Sechs der zehn Schüler*innen geben an, einen Migrationshintergrund zu haben. Signifikante

Tab. 7 Leistungen der Schüler*innen und Bildungshintergrund in den Profilen

\begin{tabular}{|c|c|c|c|c|c|c|}
\hline & $\begin{array}{l}\text { Englisch } \\
\text { Test MW } \\
\text { (SD) }\end{array}$ & $\begin{array}{l}\text { Mathe Test } \\
\text { MW (SD) }\end{array}$ & $\begin{array}{l}\text { Note Eng- } \\
\text { lisch MW } \\
\text { (SD) }\end{array}$ & $\begin{array}{l}\text { Note Ma- } \\
\text { the MW } \\
\text { (SD) }\end{array}$ & $\begin{array}{l}\text { Bücher im } \\
\text { HH MW } \\
\text { (SD) }\end{array}$ & $N$ \\
\hline Profil 1 & $-0,22(1,64)$ & $\begin{array}{l}-0,10 \\
(1,30)\end{array}$ & $2,60(1,58)$ & $2,00(1,05)$ & $3,20(1,48)$ & $\begin{array}{l}10 \\
(8,4 \%)\end{array}$ \\
\hline Profil 2 & $0,28(1,35)$ & $0,07(1,34)$ & $2,13(0,89)$ & $2,57(0,99)$ & $2,75(1,28)$ & $\begin{array}{l}52 \\
(43,7 \%)\end{array}$ \\
\hline Profil 3 & $-0,04(1,27)$ & $\begin{array}{l}-0,37 \\
(1,12)\end{array}$ & $2,18(1,00)$ & $2,40(0,87)$ & $2,79(1,28)$ & $\begin{array}{l}57 \\
(47,9 \%)\end{array}$ \\
\hline $\begin{array}{l}p(\mathrm{P} 1 \\
\text { vs. P2) }\end{array}$ & 0,39 & 0,71 & 0,19 & 0,14 & 0,32 & - \\
\hline $\begin{array}{l}p(\mathrm{P} 1 \\
\text { vs. P3) }\end{array}$ & 0,70 & 0,58 & 0,44 & 0,28 & 0,43 & - \\
\hline $\begin{array}{l}p(\mathrm{P} 2 \\
\text { vs. P3) }\end{array}$ & 0,21 & 0,07 & 0,80 & 0,35 & 0,86 & - \\
\hline
\end{tabular}


Unterschiede zu den anderen zwei Profilen in der Schulleistung, den Schulnoten und den Büchern im Haushalt können wir nicht finden. Dies ist sicherlich auch auf die kleine Stichprobe zurückzuführen. Ungeachtet des Signifikanztests berichten die Schüler*innen in Profil 1, dass sie in Mathematik die besten und in Englisch die schlechtesten Noten der drei ermittelten Profile erhalten. Die Testleistungen in Englisch sind am geringsten, in Mathematik zwischen Profil zwei und drei zu verorten (Tab. 7). Anders formuliert, kann festgehalten werden, dass selbst wenn - auf Grund der kleinen Stichprobe - der Signifikanztest nachrangig behandelt werden würde, die Schüler*innen im Profil 1 weder besonders ,gut ' noch besonders ,schlecht" sind. Es bestehen hier keine Auffälligkeiten im Vergleich zu den Schüler*innen anderer Profile, sodass nicht davon ausgegangen werden kann, dass sie keine Hilfe aufgrund guter Schulleistungen erhalten.

\subsubsection{Profil 2}

Schüler*innen, die dem zweiten Profil ( $N=52$ Schüler*innen | 43,7\%) zugeordnet werden, erfahren insgesamt betrachtet eine gelegentliche Unterstützung durch das Personal in der Hausaufgabenbetreuung. Sie geben an, dass die Lehrkräfte bzw. die Betreuer*innen eher nicht so genau wissen, was sie gut bzw. weniger gut können. Dafür sind die Zustimmungswerte in den Variablen höher, die auf die Hilfe bei der Aufgabenbearbeitung abzielen (Abb. 2: geben mir Hinweise, helfen mir Fehler zu entdecken, können gut erklären). Auch diese Schüler*innen finden sich (fast zur Hälfte) in Hausaufgabenbetreuungs- $(48,1 \%)$ und Lernzeitschulen $(51,9 \%)$ wieder. Auffällig ist jedoch, dass deutlich mehr Mädchen (61,5\%) diesem Profil angehören als Jungs (38,5\%). 63,5\% der Schüler*innen haben einen Migrationshintergrund. Wie bereits bei Profil 1 können keine signifikanten Unterschiede der Schüler*innen, in der Schulleistung, den Schulnoten oder den Büchern im Haushalt gefunden werden (Tab. 7).

\subsubsection{Profil 3}

Schüler*innen des dritten Profils sind mit 47,9\% (N=57 Schüler*innen) die größte Gruppe und berichten insgesamt die höchste Unterstützung. Besonders positiv schätzen sie die Hinweise der Lehrkräfte bzw. Betreuer*innen ein, die Zeit für Antworten sowie die Erklärungen bei Fragen (Abb. 2). Die Schüler*innen des dritten Profils kommen zu zwei Dritteln deutlich häufiger in den Schulen mit Lernzeiten vor, finden sich jedoch insgesamt an jeder der fünf Schulen. 56\% der Schüler*innen im Profil 3 geben an, dass sie Jungen sind und $63 \%$ der Schüler*innen haben einen Migrationshintergrund.

\subsection{Hilfestellungen aus Sicht der Schüler*innen}

Um die Fragen zu beantworten, wie Schüler*innen über die Hilfestellungen berichten, sie diese erleben und inwiefern die Hilfe durch die Lehrkräfte oder die Betreuer*innen als Unterstützung bei der Aufgabenerledigung wahrgenommen wird, nehmen wir Bezug auf unsere qualitativen Befunde. Basierend auf den Protokollen 
der Datenerhebungen, die Schüler*innen-Identifikationsnummern enthalten, und der Verknüpfung mit den quantitativen Daten können wir die ermittelten Profile den Schüler*innen in den jeweiligen Gruppendiskussionen zuordnen. In der ersten ausgewählten Gruppendiskussion einer Lernzeitschule nahmen Schüler*innen teil, die wir den Profilen 2 und 3 zuordnen konnten. Die drei Schüler*innen in der zweiten Gruppendiskussion können dem Profil 2 und ein*e Schüler*in dem Profil 1 zugeordnet werden. Keine der insgesamt neun Gruppendiskussionen lässt sich einem homogenen Profil zuordnen.

\subsubsection{Lernzeitschule - Nicht-Warten auf Hilfestellung (Profil 2 und 3)}

Die erste ausgewählte Sequenz greift thematisch das Warten auf Hilfestellung für Schüler*innen in der Lernzeit auf und veranschaulicht unterschiedliche Perspektiven zwischen den Schüler*innen und der Interviewerin darauf:

Iw: Müsst ihr denn lange warten, wenn ihr Hilfe braucht, //dann auch//

Sm1: //<Nein (schüttelt mit dem Kopf) $>/ /$

Sm2: //<Nein (schüttelt mit dem Kopf) $>/ /$

Iw: also <wenn du sagst (zeigt in Richtung Sm2)> da kommen alle rein. //Müsst

ihr/ also dauert es dann manchmal?//

Sw1: //Manchmal vielleicht wenn sehr viele// Leu/ Fra/ Fragen haben, dann.

In der Gruppendiskussion greift die Interviewerin eine Problematik (des Unterrichts) auf, die auch in der Literatur diskutiert wird (s. Forschungsstand), wie es einer Lehrkraft bzw. einer*m Betreuer*in gelingt, mehreren Schüler*innen - die an unterschiedlichen Aufgaben arbeiten und dabei ggf. parallel Fragen zur Aufgabenerledigung aufkommen - zeitgleich zu helfen. Über das Verb „müssen“ geht sie zunächst von einem Zwangscharakter aus und nimmt implizit an, dass Schüler*innen auf jeden Fall warten müssen, wenn diese „Hilfe brauchen“. Die Frage enthält also einen suggestiven Charakter. In der Frage bezieht sich das Warten nicht unmittelbar auf etwas Ersehntes, wie das Unterrichtsende, sondern beinhaltet einen Modus des sich Bereithaltens. Das Warten ist in diesem Kontext seitens der Interviewerin mit Inaktivität verbunden. Sie geht davon aus, dass die Schüler*innen auf jeden Fall Hilfe benötigen und folglich auch Warten müssen. Das Nicht-Warten ist in der Formulierung der Interviewerin keine Option, sondern es geht direkt um die Dauer des Wartens, was über den Sprechakt „lange Warten“ artikuliert wird. Darin ist zugleich eine negative Konnotation enthalten, die durch das vorangestellte „lange“ verstärkt wird. Eine lange Zeitspanne zielt dabei auf eine subjektive Einschätzung ab, was wiederum zu dem Zusatzwort ,denn“ passt. Dieses nimmt der Frage die Strenge und weist auf ein emphatisches Mitfühlen hin, um sich den Schüler*innen nahezustellen und Interesse an ihrem Erleben anzuzeigen. Die Bedürftigkeit nach Hilfe wird in dieser Sequenz zugeschrieben und nicht das Wollen von Hilfe; denn wer Hilfe möchte, aber nicht benötigt, wartet nicht lange. Hilfebrauchen steht auch dahingehend für Bedürftigkeit, da es ohne Hilfe nicht weitergeht und ein langes Warten für kleine Probleme nicht in Kauf genommen wird.

Parallel dazu antworten zwei Schüler und verneinen die Frage. Dieses „Nein“ kann sowohl in Bezug auf das ,lange Warten“ - im Sinne von ,nein, wir müssen 
nicht lange warten " - gelesen werden, als auch als , nein, wir brauchen keine Hilfe . Außerdem kann das Nein aber auch aus Schüler*innenperspektive bedeuten: ich mach in der Zeit etwas anderes/ich organisiere mich selbständig um ' und warte nicht (lang). Das Nein wird von beiden Schülern nonverbal durch paralleles Kopfschütteln unterstrichen.

Auf diese Antworten folgt eine Irritation der Interviewerin (Verkettung von Füllwörtern „dann auch“) und die Antwort der Schüler scheint unpassend, da für eine weitere Spezifizierung ausgeholt wird (,also“). Es bleibt jedoch offen, worum es geht: das Hilfebekommen zu spezifizieren oder das Warten. Das „,nein“ der Schüler schließt den - noch nicht in Gang gekommenen - Diskurs über Hilfe und Warten, während das ,also“ der Interviewerin ihn beleben möchte. Diese Lesart, wird vor dem Hintergrund des Anschlusses ,wenn du sagst“ plausibel. Dabei adressiert sie einen der verneinenden Schüler (,du“) anblickend und eröffnet die Option für eine Korrektur der Schülerantworten. Mit „wenn du sagst da kommen alle rein“ nimmt sie auf die vorherige Passage Bezug. Dabei geht es um eine konkrete Situation (,da“) oder einen virtuellen Ort, wie das Betreten eines Raumes, und dieser Sprechakt plausibilisiert die Lesart, dass die Interviewerin mit dem Nein als Antwort nicht ,zufrieden“ ist, da sie eine abstrakte Erzählung (,da kommen alle rein“) beginnt, die spezifiziert, worauf die Frage eigentlich abzielt und auf diese Weise nochmal nachhakt.

Auch dieser Sprechakt wird jedoch abgebrochen und die Suche nach weiteren Antworten wird über „Müsst ihr/ also dauert es dann manchmal?“ erneut aufgegriffen. Die Interviewerin fragt indirekt danach, wie es funktioniert, wenn alle Schüler*innen Hilfe benötigen. Dabei ist der Ausgangspunkt, dass Hilfebenötigen (und Bekommen) essenziell für die Lernzeit ist und greift die mögliche Herausforderung des „lange Wartens“ auf. Es geht demnach thematisch eher um die Frage des Organisierens individueller Hilfen und wie dies im Falle möglicher zeitlicher Engpässe gehandhabt wird, zielt eigentlich auf eine subjektive Einschätzung aus Schülerperspektive. Entsprechend erhält sie zwar subjektive Antworten, die aber nicht zufriedenstellend die, eigentliche" Frage zum Organisieren individueller Hilfen beantworten. Dies zeigt sich im impliziten Erstaunen, wieso die Schüler*innen nicht lange warten müssen. Während die Interviewerin noch bei ihrer Frage (wieso müsst ihr nicht lange warten) ist, stellt hingegen für die Schüler*innen das Warten und die Dauer des Wartens kein Problem dar. Daran verdeutlichen sich zwei unterschiedliche Sichtweisen auf das Warten, da für die Interviewerin Warten in Kontrast zu ,wertvoller Lernzeit" steht und für die Schüler*innen die Lernzeit so aber in Ordnung ist bzw. eine Lernzeit mit Warten kein ,Drama' darstellt und sie andere Strategien haben oder auch Relevanzen setzen.

Die Intervention der Interviewerin bringt dann dennoch eine ,erhoffte' Frageintention einer Schülerin hervor: „Manchmal vielleicht wenn sehr viele// Leu/ Fra/ Fragen haben, dann“. Zugleich kommt dies jedoch nur ,manchmal“ und ,vielleicht“ in Situationen vor, wenn sehr viele Fragen haben und dies scheint eher auf eine Besonderheit (langes Warten) statt Regelhaftigkeit seitens der Schüler*innen zu verweisen. Die in der Literatur beschriebene Problematik der zeitgleichen Organisation individuellen Lernens ist in dieser Sequenz aus Perspektive der Schüler*innen - im Kontrast zur Perspektive der Interviewerin - also unbedeutend. 


\subsubsection{Hausaufgabenbetreuung - Hilfe durch die Betreuer*innen bzw. Lehrkräfte (Profil 1 und 2)}

Die zweite Sequenz ist einer Gruppendiskussion in einer Schule mit Hausaufgabenbetreuung entnommen. Auch hier wird gezielt nach der Hilfe durch die Betreuer*innen gefragt.

Iw: Ähm, die nächste Frage, da interessiert mich, ob euch die Betreuerin oder eure Lehrer in der Hausaufgabenbetreuung helfen?

Sm1: (Ne?)

Sw2: //Ähm//

Iw: //(lacht)//

Sw2: <Jaein (lacht; zieht das Wort in die Länge)>, würde ich mal so sagen, also ja und nein, weil manchmal helfen die uns nicht, die sagen nur so (3) (räuspert sich), also (lacht) die sagen nur so: „Ja, <guckt mal genauer hin, also lies mal genauer (gestikuliert) $>$ und dann findest du es vielleicht heraus", und dann gehen die auch immer weg und gehen zu einem anderen, die gucken einfach manchmal nur drüber und <manche (zieht das Wort in die Länge) >, also, Betreuerinnen da (3), oder Lehrer, helfen uns wirklich auch

Thematisch lenkt die Interviewerin ihre Frage auf die Hilfestellung für Schüler*innen durch die Betreuer*innen. Die Frage nach der Hilfe wird zunächst verneint. Da dies fragend gesagt wird, wirkt diese Antwort jedoch nicht ganz überzeugt, sondern eher überlegend. Das ,ähm“ der Schülerin kann intervenierend verstanden werden, aber auch als ausholend für eine längere Redesequenz. Nach dem Lachen der Interviewerin - dessen Bedeutung ambivalent bleibt - führt die Schülerin mit einem ,Jaein“ ihren Beitrag fort. Somit wird deutlich, dass das Ähm hier als korrigierender Einwand zu Schüler Sm1 zu verstehen ist. Das Jaein ist in diesem Sinne ein ,kommt darauf an“, wobei die Schülerin Sw2 mit „würde ich mal so sagen“ ihren Einwand relativierend beginnt. Die Vorsicht in dem Sprechakt tritt klar hervor und ermöglicht es ihr zugleich die Aussage zu revidieren, sollte hier ein Widerspruch folgen. Die Verwendung des Konjunktives widerspricht dabei der Tatsache, dass sie es bereits ausgesprochen hat. Möglich ist, dass sie dadurch keine zu starke Gegenposition einnehmen möchte. Mit dem darauffolgendem ,ja und nein“ verdeutlicht sie dies nochmal.

Die Begründung für ihr Nein folgt mit der nun anschließenden Absage (,,weil manchmal helfen die uns nicht“), wobei nicht zwischen „Betreuerin“ und „Lehrern“ differenziert wird. Die Quantifizierung „manchmal“ zeigt zugleich an, dass dies nicht die Regel darstellt. Hieran anschließend imitiert sie die Betreuenden bzw. Lehrkräfte: „die sagen nur so“. Dieser Sprechakt drückt zugleich aus, dass diese zwar etwas unternehmen, dies aber keine Hilfe darstellt. Eine, richtige ' Hilfe ist vielmehr an ein Tun geknüpft, ,sagen“ suggeriert hingegen, dass nur geredet, aber eben nichts getan wird. Das „nur“ verdeutlicht, dass diese Art der Hilfestellung für die Schülerin zu wenig ist und nicht reicht. Die Imitation führt sie fort mit dem doppelten Verweis auf genaueres Lesen und die Chance, es selbst zu lösen (,dann findest du es vielleicht heraus“). Im Kontext des Fragebogen-Items „geben 
mir Hinweise wie ich eine Aufgabe gut lösen kann“ würde dazu keine Zustimmung bestehen.

Zugleich geht Sw2 auf eine Reaktion der Betreuerin bzw. der Lehrkräfte ein, die unterstellen, dass die Schüler*innen - sie spricht hier allgemein und nicht auf sich bezogen - sich noch nicht hinreichend bemüht hätten. Die spezifische Hilfeanfrage wird in diesem Sinne zurückgewiesen. Diese Zurückweisung der Hilfebedürftigkeit mit dem spezifischen Auftrag etwas zu tun (genauer hingucken, lesen) und der gleichzeitigen Unterstellung, dies nicht bereits getan zu haben, wird daher nicht als Hilfe wahrgenommen. Auffällig ist, dass durch die Relativierung ,vielleicht“ die Möglichkeit besteht, dass die Schüler*innen, selbst wenn sie sich größte Mühe geben, es nicht oder nicht unbedingt - also ohne Hilfestellung - herausfinden (können). Das Medium der Aufgabe, die möglicherweise zu schwer oder nicht gut gestellt sein könnte, wird nicht in Frage gestellt, sondern die beispielhafte Hilfeanfrage wird an die Schüler*innen readressiert, sich mehr anzustrengen und es nochmal zu versuchen.

Zur Zurückweisung der Hilfeanfrage und zur Unterstellung, sich nicht genug angestrengt zu haben, kommt noch die Kritik der Kurzweiligkeit der Hilfe hinzu und keinen Ansprechpartner mehr zu haben (immer weggehen, zu anderen). „Immer“ deutet hier auf eine Regelhaftigkeit hin. Die sich durchziehende Verwendung von „die“ weist zusätzlich auf die negative Konnotation der Aussage der Schülerin hin, da ,die“ nicht benannt oder konkretisiert wird. Dies bestärkt sich auch in der darauffolgenden Kritik an der Art der Hilfe (,,einfach manchmal nur drüber“ gucken). Hier wird weiterhin unpersönlich und (aus dem Kontext geschlossen) negativ über ,die“, die nicht helfen, gesprochen. Das kritisierte Drüber gucken ist mit Oberflächlichkeit verknüpft, was durch den Zusatz ,einfach“ verstärkt wird. So entsteht der Eindruck, dass hier ,nur so getan wird, als ob` geholfen wird. Es wird lediglich über das Medium geschaut, jedoch nicht persönlich mit Blick auf den individuellen Hilfebedarf. ,Ideale' Hilfe hingegen wäre persönlich und nicht so oberflächlich.

Abschließend erwähnt die Schülerin jedoch das positive Beispiel, dass manche auch, wirklich' helfen und benennt allgemein die Betreuer*innen und Lehrer. Schlussendlich wird deutlich, dass die Ausnahme darin besteht, dass ein kleiner Teil der pädagogischen Akteur*innen (,manche“) wirklich hilft, im Unterschied zum Normalfall, in dem keine Hilfe wahrgenommen wird. Durch ,wirklich“ kommt erneut zum Ausdruck, dass es eine Hilfe gibt, die zunächst als solche erscheint, aber aus Schüler*innenperspektive nicht als ,echte' Hilfe wahrgenommen wird und offenbar auch ,richtige ' Hilfe, die jedoch nicht weiter ausgeführt wird.

Hierrüber wird zugleich auf ein Problem der standardisierten Abfrage im Fragebogen verwiesen, die sich allgemein auf die Hilfe oder Unterstützung bezieht. Die Schüler*in erlebt entsprechend bei einigen Betreuer*innen keine Unterstützung, bei anderen aber durchaus. Dieser Grad der Differenzierung wird im Fragebogen jedoch nicht berücksichtigt. 


\section{Diskussion}

Zusammenfassend zeigen die hier präsentierten Ergebnisse, dass die Hausaufgabenbetreuung und Lernzeiten von unterschiedlichen Schüler*innen besucht werden und hier keineswegs - wie erwartet - Schüler*innen, die entweder schlechtere Schulleistungen zeigen und/oder zuhause nicht auf entsprechende Hilfe der Eltern zurückgreifen können, überrepräsentiert sind. Dies steht im Widerspruch zu den Befunden von Tillack et al. (2015), die zeigen, dass Schüler*innen mit Migrationshintergrund in der Hausaufgabenbetreuung überrepräsentiert sind, während die hier präsentierten Ergebnisse tendenziell auf das Gegenteil bzw. keine Unterschiede hinweisen. Die Befunde von Tillack et al. (2015) beziehen sich jedoch auf die Grundschule und nutzen ältere Daten. Dies darf durchaus auch als positiv eingeordnet werden, da somit die Teilnahme an Hausaufgabenbetreuung oder Lernzeiten nicht stigmatisierend für bestimmte Schüler*innengruppen ist, wie etwa Förderangebote (Sauerwein und Heer 2020). Zudem erhöht sich die Teilnahme am Ganztag insgesamt (KMK 2020) und führt über kurz oder lang zu einer Normalisierung der Nutzung von Ganztagsangeboten. Die Unterschiede zu den Ergebnissen von Tillack et al. (2015) können jedoch auch auf einen konzeptionellen Wandel der Ganztagsschulen zurückgeführt werden, indem Lernzeiten für alle Schüler*innen statt Hausaufgabenbetreuung für eine Teilgruppe angeboten werden (s. hierzu Brisson et al. 2021; Brisson und Theis 2020; Nordt 2020).

Die Differenzierung in drei Profile der Hilfegewährung in der Hausaufgabenbetreuung und den Lernzeiten, mit den hieran anschließenden qualitativen Rekonstruktionen erlaubt einen tiefergehenden Einblick: $48 \%$ der Schüler*innen konnten einem Profil zugeordnet werden mit hohen Zustimmungsraten bezüglich der Hilfe durch die Betreuer*innen, $44 \%$ einem Profil mit einer mittleren Zustimmungsrate und lediglich $8 \%$ der Schüler*innen berichten von geringer Hilfestellung. Substanzielle Unterschiede zwischen den Profilen hinsichtlich sozialer Herkunft und Schulleistungen finden wir nicht.

Die hier anschließenden Sequenzen deuten darauf hin, dass in der Literatur antizipierte Probleme des individualisierten Unterrichts, insbesondere bei der Unterstützung der Schüler*innen bei unterschiedlichen Individualanliegen (s. a. Breidenstein et al. 2017), aus Perspektive der Schüler*innen kein Problem darstellen. Die Gründe hierfür können wir letztlich nur antizipieren. So ist es durchaus möglich, dass Lernen anders gestaltet wird, Schüler*innen in Wartezeiten andere Aufgaben erledigen, ihre Mitschüler*innen fragen oder die Aufgaben entsprechend gut erstellt sind bzw. vorbereitet oder aufgegeben werden, so dass Schüler*innen diese selbständig bearbeiten können.

Als ebenso bedeutsam erweist sich die Verknüpfung von quantitativen und qualitativen Daten. Die notwendigerweise in Fragebögen abstrakte verallgemeinernde Abfrage nach Hilfestellung in den Hausaufgaben bringt Unschärfen mit sich, wie die Analyse der zweiten Sequenz verdeutlicht. Das Personal in der Hausaufgabenbetreuung scheint unterschiedlich zu agieren und Schüler*innen nehmen dies wahr. Die Frage- und Antwortformate des Fragebogens erlauben hier aber keine entsprechende Differenzierung. Durch die Verknüpfung der Gruppendiskussionen mit den Profilen konnten wir jedoch die wahrgenommene Hilfestellung vertiefend betrachten. Aus 
Schüler*innenperspektive zeigt sich, dass die Betreuer*innen ihre Anfragen nach Hilfe nicht ernst genug und sich entsprechend zu wenig Zeit nehmen. In diesem Sinne darf die fortschreitende Entgrenzung der Schule - die weit mehr umfasst als den Topos Hausaufgaben - auch kritisch hinterfragt werden. Exemplarisch können hier die zeitlichen Fixpunkte der Lernzeiten oder Hausaufgabenbetreuung, weniger Rückzugsmöglichkeiten oder die Lautstärke beim Bearbeiten der Aufgaben in der Gruppe aufgeführt werden. Auch die Sinnhaftigkeit einer Hausaufgabenbetreuung mit Blick auf Lernprozesse ist zu hinterfragen, wenn dort nicht entsprechend kompetent ausgebildetes Personal tätig ist, welche den Schüler*innen auch die notwendige Hilfestellung geben kann. Hier könnten entsprechende Qualitätsstandards der pädagogischen Praxis zielführend sein, so dass diese Zeiten auch für Schüler*innen zu „Lern-Zeiten“ - im semantischen Sinne des Wortes - werden und nicht lediglich beaufsichtigte Zeiten.

Die vorgelegten Analysen und Daten weisen jedoch auch Begrenzungen auf. Zunächst wird in StEG-S nicht zwischen Lernzeiten und der Hausaufgabenbetreuung differenziert. Beide Formate sind zumindest programmatisch zu unterscheiden: Die Teilnahme an Lernzeiten ist obligatorisch, während Hausaufgabenbetreuung ein frei wählbares Angebot darstellt. Auch finden Lernzeiten zumeist im Klassenkontext mit Lehrkräften statt, während bei der Hausaufgabenbetreuung auch anderes Personal eingesetzt wird (Brisson und Theis 2020; Dohrmann et al. 2021; Maschke et al. 2019). Die Datengrundlage erlaubt hier keine Unterscheidung, entweder, weil dies nicht differenziert erfasst wurde - wie in StEG-S - oder andernfalls die Stichprobe zu gering ist - wie in StEG-Tandem. Ebenso geben unsere Ergebnisse keinen Aufschluss darüber, weshalb das Warten auf Hilfe für die Schüler*innen unbedeutend ist oder inwiefern das mit bestimmten pädagogischen Akteur*innen verbunden wird. Dies darf auch als Anregung für weitere Analysen der Praxen der Hilfestellung in Lernzeiten und der Hausaufgabenbetreuung genutzt werden. Die Verknüpfung zwischen den Aufgaben, die zu bearbeiten sind, und der (erfragten, benötigten) Hilfestellung gilt es in zukünftigen Arbeiten vertiefend zu betrachten. Gerade wenn Aufgaben gut erstellt, besprochen und aufgegeben sind, basierend auf dem individuellen Lernstand der Schüler*innen, könnten diese im Idealfall die Aufgaben ohne Hilfe selbständig bearbeiten. Dass für die Wirkung von Hausaufgaben die Qualität dieser entscheidend ist, belegen vorliegende Studien (Trautwein et al. 2006). Diese Annahme bestätigen auch unsere Daten: Schüler*innen in Profil 3 stimmen der Aussage „Unser*e Mathe-Lehrer*in erklärt deutlich, wie wir unsere Hausaugaben/Lernzeit-Aufgaben bearbeiten sollen“ im Mittel deutlich häufiger zu als die Schüler*innen der anderen zwei Profile (Profil 3 MW: 3,62 SD: 0,65 | Profil 2 MW: 3,22 SD: 0,92 | Profil 1: MW: 2,90 SD: 0,99), wobei signifikante Unterschiede von Profil 3 zu den Profilen 2 und 1 bestehen (zwischen Profil 1 und 2 bestehen keine signifikanten Unterschiede). Dies gilt es in weiteren Studien zu vertiefen, die ebenso die Erwartungen der Schüler*innen und Betreuenden an „Hilfestellung“ genauer analysieren (Wie ist der Umgang miteinander bei der Bearbeitung? Welche Hilfestellung wird erfragt, welche gegeben? Welchen Einfluss auf die seitens der Schüler*innen wahrgenommene Unterstützung hat der Habitus der Betreuenden?) sowie auch unterschiedliche Konzepte der Umsetzung miteinander systematisch vergleichen. Eine Akteurszentrierung statt einer Fokussierung auf die 
Organisation Schule oder die Funktionalität der Hausaufgaben-Settings erscheint hier basierend auf unseren Ergebnissen vielversprechend.

Schließlich sind Latente Profilanalysen ein exploratives Verfahren, welches sich für die Fragestellung des Beitrags anbietet, aber in weiteren Arbeiten zu verdichten wäre und auch in theoretische bzw. konzeptionelle Überlegungen münden sollten, die wiederum geprüft werden können.

Resümierend zeigen unsere Ergebnisse, dass der Perspektive der Schüler*innen auf Lernzeiten bzw. die Hausaufgabenbetreuung mehr Raum gegeben werden sollte. Ebenso ermöglicht dies latent vorhandene Annahmen, wie die, dass unterschiedliche Individualanliegen der Schüler*innen automatisch zu Phasen des Wartens führen (s. Forschungsstand und Gruppendiskussion 1), zu hinterfragen. Gerade die Verknüpfung von qualitativen und quantitativen Daten ist hier gewinnbringend und anregend für weitere Forschungsfragen und Analysen.

Förderung Die diesem Beitrag zugrundeliegenden Forschungsprojekte StEG-S und StEG-Tandem wurden aus Mitteln des Bundesministeriums für Bildung und Forschung (BMBF) gefördert. Die Sekundärauswertung wurde finanziert vom Zentrum für Schul-, Bildungs- und Hochschulforschung (ZSBH) der Johannes Gutenberg-Universität Mainz. Die Verantwortung für den Inhalt dieser Veröffentlichung liegt bei den Autor*innen.

Funding Open Access funding enabled and organized by Projekt DEAL.

Open Access Dieser Artikel wird unter der Creative Commons Namensnennung 4.0 International Lizenz veröffentlicht, welche die Nutzung, Vervielfältigung, Bearbeitung, Verbreitung und Wiedergabe in jeglichem Medium und Format erlaubt, sofern Sie den/die ursprünglichen Autor(en) und die Quelle ordnungsgemäß nennen, einen Link zur Creative Commons Lizenz beifügen und angeben, ob Änderungen vorgenommen wurden.

Die in diesem Artikel enthaltenen Bilder und sonstiges Drittmaterial unterliegen ebenfalls der genannten Creative Commons Lizenz, sofern sich aus der Abbildungslegende nichts anderes ergibt. Sofern das betreffende Material nicht unter der genannten Creative Commons Lizenz steht und die betreffende Handlung nicht nach gesetzlichen Vorschriften erlaubt ist, ist für die oben aufgeführten Weiterverwendungen des Materials die Einwilligung des jeweiligen Rechteinhabers einzuholen.

Weitere Details zur Lizenz entnehmen Sie bitte der Lizenzinformation auf http://creativecommons.org/ licenses/by/4.0/deed.de.

Interessenkonflikt M. Sauerwein und P. Rother geben an, dass kein Interessenkonflikt besteht.

\section{Literatur}

Altermann, A., Börner, N., Lange, M., Menke, S., Steinhauer, R., \& Tabel, A. (2016). Bildungsbericht Ganztagsschule NRW 2016. BiGa NRW. Dortmund: Eigenverlag Forschungsverbund DJI/TU Dortmund.

Auer, M., Gruber, G., Mayringer, H., \& Wimmer, H. (2005). Salzburger Lese-Screening für die Klassenstufe 5-8 (SLS 5-8). Bern: Huber.

Beher, K., Haenisch, H., Hermens, C., Nordt, G., Prein, G., \& Schult, U. (Hrsg.). (2007). Die offene Ganztagsschule in der Entwicklung. Empirische Befunde zum Primarbereich in Nordrhein-Westfalen. Weinheim: Juventa.

BMFSFJ (2021). Neunter Familienbericht: Eltern sein in Deutschland - Ansprüche, Anforderungen und Angebote bei wachsender Vielfalt. Berlin: BMFSFJ.

Breidenstein, G., \& Rademacher, S. (2013). Vom Nutzen der Zeit. Beobachtungen und Analysen zum individualisierten Unterricht. Zeitschrift für Pädagogik, 59, 336-356. 
Breidenstein, G., \& Rademacher, S. (2017). Einleitung. In G. Breidenstein \& S. Rademacher (Hrsg.), Individualisierung und Kontrolle (S. 1-16). Wiesbaden: Springer VS.

Breidenstein, G., Dorow, S., Menzel, C., \& Rademacher, S. (2017). Die Organisation individualisierten Unterrichts. In G. Breidenstein \& S. Rademacher (Hrsg.), Individualisierung und Kontrolle (S. 17-74). Wiesbaden: Springer VS.

Brisson, B. M., \& Theis, D. (2020). Traditionelle Hausaufgaben oder integrierte Lernzeiten? Ein Vergleich der Erfahrungen und Leistungsentwicklungen von Schülerinnen und Schülern mit unterschiedlichen Übungsformaten. Psychologie in Erziehung und Unterricht, 67(4), 294-312.

Brisson, B., Heyl, K., Sauerwein, M., Theis, D., \& Fischer, N. (2017). Leitfaden StEG-Tandem. Konzeptuelle Weiterentwicklung von Hausaufgabenbetreuung und Lernzeiten. Studie zur Entwicklung von Ganztagsschulen

Brisson, B., Heyl, K., Sauerwein, M., Theis, D., Fischer, N., \& Klieme, E. (2021). StEG-Tandem: Schulentwicklungsprojekt und Evaluationsstudie. In H. G. Holtappels, N. Fischer, S. Kielblock, B. Arnoldt \& J.M. Gaiser (Hrsg.), Individuelle Förderung an Ganztagsschulen (S. 96-104). Weinheim: Beltz Juventa.

Brisson, B., Sauerwein, M., Heyl, K., \& Theis, D. (2019). StEG-Tandem: Eine Schulentwicklungsstudie zur Einführung von kooperativen Lernformen in Hausaufgabenbetreuung bzw. Lernzeiten an Ganztagsschulen Hintergrund, Konzeption und erste Ergebnisse. In S. Maschke, G. Schulz-Gade \& L. Stecher (Hrsg.), Jahrbuch Ganztagsschule. 2019/20: Hausaufgaben und Lernzeiten pädagogisch sinnvoll gestalten. Aktuelle Entwicklungen und Diskussionslinien (S. 121-138). Frankfurt a. M.: Debus Pädagogik.

Budde, J. (2012). Didaktische Regime - Zettelwirtschaft zwischen Differenzstrukturen, Homogenisierung und Individualisierung. In J. Budde (Hrsg.), Unscharfe Einsätze. (Re-)Produktion von Heterogenität im schulischen Feld (S. 169-186). Wiesbaden: Springer VS.

Chiapparini, E. (2019). Erweiterte Lernzeiten und ambivalente Förderung des Wohlbefindens der Kinder an Tagesschulen in Basel-Stadt. In S. Maschke, G. Schulz-Gade \& L. Stecher (Hrsg.), Jahrbuch Ganztagsschule. 2019/20: Hausaufgaben und Lernzeiten pädagogisch sinnvoll gestalten. Aktuelle Entwicklungen und Diskussionslinien (S. 200-211). Frankfurt a. M.: Debus Pädagogik.

Dohrmann, J., Brisson, B., \& Kielblock, S. (2021). Qualität und Wirkung von Peer Mentoring aus Sicht der Schüler*innen. In H. G. Holtappels, N. Fischer, S. Kielblock, B. Arnoldt \& J. M. Gaiser (Hrsg.), Individuelle Förderung an Ganztagsschulen (S. 105-119). Weinheim: Beltz Juventa.

Dumont, H. (2019). Neuer Schlauch für alten Wein? Eine konzeptuelle Betrachtung von individueller Förderung im Unterricht. Zeitschrift für Erziehungswissenschaft, 22(2), 249-277.

Gaiser, J.M., \& Kielblock, S. (2019). Von Hausaufgaben zu rhythmisierten Lernzeiten. Ganztagsschulentwicklung am Beispiel einer längsschnittlichen Fallstudie. Zeitschrift für Bildungsforschung, 9(2), $159-175$.

Gaiser, J. M., Sauerwein, M., \& Kielblock, S. (2020). Außerunterrichtliche Lern- und Förderarrangements an Ganztagsschulen: Bundesweite Trendanalysen und vertiefende Fallstudien. Psychologie in Erziehung und Unterricht, 67(4), 243-261.

Gängler, H., \& Markert, T. (Hrsg.). (2011). Vision und Alltag der Ganztagsschule. Die Ganztagsschulbewegung als bildungspolitische Kampagne und regionale Praxis. Weinheim: Beltz Juventa.

Giegler, H., \& Rost, J. (1993). Typenbildung und Responsesets beim Gießen-Test: Clusteranalyse versus Analyse latenter Klassen. Zeitschrift für Differentielle und Diagnostische Psychologie, 14(3), $137-152$.

Gollwitzer, M. (2012). Latent-Class-Analysis. In H. Moosbrugger \& A. Kelava (Hrsg.), Testtheorie und Fragebogenkostruktion (S. 295-323). Berlin: Springer Berlin Heidelberg.

Grimm, W., \& Schulz-Gade, G. (2017). Übungs- und Lernzeiten an der Ganztagsschule. Ein Praxisleitfaden zur Integration von Hausaufgaben in den Ganztag (1. Aufl.). Berlin: Wochenschau Verlag.

Hess, A. (2020). Disziplin und Leistung im Alltag einer zweiten Grundschulklasse. Eine ethnographische Studie. Wiesbaden: Springer VS.

Holtappels, H., Lossen, K., Osadnik, F., Rollett, W., Tillmann, K., \& Wutschka, K. (2019). StEG-Lesen. In StEG-Konsortium (Hrsg.), Individuelle Förderung: Potenziale der Ganztagsschule (S. 26-35).

Kaplan, D., \& Keller, B. (2011). A note on cluster effects in latent class analysis. Structural Equation Modeling: A Multidisciplinary Journal, 18(4), 525-536.

Killus, D., \& Tillmann, K.-J. (Hrsg.). (2017). Eltern beurteilen Schule - Entwicklungen und Herausforderungen. Ein Trendbericht zu Schule und Bildungspolitik in Deutschland. Die 4. JAKO-O Bildungsstudie. Münster: Waxmann.

Klieme, E. (2019). Unterrichtsqualität. In M. Gläser-Zikuda, M. Harring \& C. Rohlfs (Hrsg.), Handbuch Schulpädagogik (1. Aufl.). Stuttgart: UTB. 
Klieme, E., \& Rakoczy, K. (2008). Empirische Unterrichtsforschung und Fachdidaktik. Outcome-orientierte Messung und Prozessqualität des Unterrichts. Zeitschrift für Pädagogik, 54(2), 222-237.

Klieme, E., \& Warwas, J. (2011). Konzepte der Individuellen Förderung. Zeitschrift für Pädagogik, 57(6), 805-818.

KMK - Sekretariat der Ständigen Konferenz der Kultusminister der Länder in der Bundesrepublik Deutschland (2020). Allgemeinbildende Schulen in Ganztagsform in den Ländern in der Bundesrepublik Deutschland - Statistik 2014 bis 2018.

Kohler, B. (2017). Hausaufgaben: Überblick und Praxishilfen für Halbtags- und Ganztagsschulen. Weinheim: Beltz.

Magidson, J., \& Vermunt, J. (2004). Latent class models. In D. Kaplan (Hrsg.), The Sage handbook of quantitative methodology for the social sciences (S. 175-198). Thousand Oaks: SAGE.

Marcus, J., Nemitz, J., \& Spieß, C.K. (2016). Veränderungen in der gruppenspezifischen Nutzung von ganztägigen Schulangeboten - Längsschnittanalysen für den Primarbereich. Zeitschrift für Erziehungswissenschaft, 19(2), 415-442.

Maschke, S., Schulz-Gade, G., \& Stecher, L. (Hrsg.). (2019). Jahrbuch Ganztagsschule. 2019/20: Hausaufgaben und Lernzeiten pädagogisch sinnvoll gestalten. Aktuelle Entwicklungen und Diskussionslinien. Frankfurt a. M.: Debus Pädagogik.

Morin, A. J., Meyer, J. P., Creusier, J., \& Biétry, F. (2016). Multiple-group analysis of similarity in latent profile solutions. Organizational Research Methods, 19(2), 231-254.

Nordt, G. (2020). Hausaufgaben - Schulaufgaben - Lernzeiten. In P. Bollweg, J. Buchna, T. Coelen \& H.U. Otto (Hrsg.), Handbuch Ganztagsbildung (S. 1045-1061). Wiesbaden: Springer VS.

Nordt, G., \& Röhner, C. (2008). Hausaufgaben in der offenen Ganztagsgrundschule - ein Beitrag zur Förderung des schulischen Lernens und der Schulqualität. Widersprüche, 110, 67-80.

Nylund, K. L., Asparouhov, T., \& Muthén, B. O. (2007). Deciding on the number of classes in latent class analysis and growth mixture modeling: a Monte Carlo simulation study. Structural Equation Modeling: A Multidisciplinary Journal, 14(4), 535-569.

Praetorius, A.-K., Klieme, E., Kleickmann, T., Brunner, E., Lindmeier, A., Taut, S., \& Charalambous, C. Y. (2020). Towards developing a theory of generic teaching quality. Zeitschrift für Pädagogik, 66, $15-36$.

Rabenstein, K., \& Podubrin, E. (2015). Praktiken individueller Zuwendung in Hausaufgaben- und Förderangeboten. Empirische Rekonstruktionen pädagogischer Ordnungen. In S. Reh, B. Fritzsche, T.S. Idel \& K. Rabenstein (Hrsg.), Lernkulturen (S. 219-263). Wiesbaden: Springer VS.

Rother, P. (2019). Studien zur ganztägigen Bildung: Sortieren als Umgang mit Bildungsbenachteiligung. Orientierungen pädagogischer Akteure in einem kooperativen Ganztags-Setting. Weinheim: Beltz Juventa.

Sauerwein, M. (2017). Qualität in Bildungssettings der Ganztagsschule: Über Unterrichtsforschung und Sozialpädagogik. Weinheim: Beltz Juventa.

Sauerwein, M., \& Heer, J. (2020). Warum gibt es keine leistungssteigernden Effekte durch den Besuch von Ganztagsangeboten? Zeitschrift für Pädagogik, 66(1), 78-201.

Sauerwein, M.N., Thieme, N., \& Chiapparini, E. (2019). Wie steht es mit der Ganztagsschule? Ein Forschungsreview mit sozialpädagogischer Kommentierung. Soziale Passagen, 11(1), 81-97.

Sauerwein, M., \& Rother, P. (i. E., angenommen). Teilhabe und Anerkennung statt Chancengerechtigkeit - eine sozialpädagogische Perspektive auf Ganztagsschule. In M. Jörgens, J. Sander \& S. Werner (Hrsg.), Lesen im Ganztag: systematische Leseförderung in systemischer Perspektive (Lesesozialisation und Medien). Weinheim: Beltz Juventa.

Souvignier, E., Förster, N., \& Schulte, E. (2014). quop: Ein Ansatz internetbasierter Lernverlaufsdiagnostik mit Testkonzepten für Lesen und Mathematik. In M. Hasselhorn (Hrsg.), Lernverlaufsdiagnostik. Tests und Trends (Bd. 12, S. 239-256). Göttingen: Hogrefe.

Souvignier, E., Trenk-Hinterberger, I., Adam-Schwebe, S., \& Gold, A. (2008). FLVT 5-6 Frankfurter Leseverständistest für 5. und 6. Klassen. Manual. Göttingen: Hogrefe.

StEG-Konsortium (2019). Ganztagsschule 2017/2018. Deskriptive Befunde einer bundesweiten Befragung. https://www.pedocs.de/volltexte/2019/17105/pdf/Ganztagsschule_2017_2018_StEG.pdf. Zugegriffen: 14. Okt. 2021.

Steiner, C. (2009). Mehr Chancengleichheit durch die Ganztagsschule? Zeitschrift für Pädagogik, 54, 81-105.

Steinmann, I., \& Strietholt, R. (2019). Effekte nachmittäglicher Bildungsangebote auf sprachliche Kompetenzentwicklung. Zeitschrift für Pädagogik, 65(2), 285-306.

Strietholt, R., Manitius, V., Berkemeyer, N., \& Bos, W. (2015). Bildung und Bildungsungleichheit an Halbund Ganztagsschulen. Zeitschrift für Erziehungswissenschaft, 18(4), 737-761. 
Tillack, C., Fischer, N., \& Kuhn, H. (2015). Hausaufgabenbetreuung in der Ganztagsschule. Soziale Unterstützung für Schülerinnen und Schüler mit Migrationshintergrund? Zeitschrift für Grundschulforschung, 8(2), 114-130.

Trautwein, U., Lüdtke, O., Schnyder, I., \& Niggli, A. (2006). Predicting homework effort: support for a domain-specific, multilevel homework model. Journal of Educational Psychology, 98(2), 438-456.

Wernet, A. (2009). Einführung in die Interpretationstechnik der Objektiven Hermeneutik (3. Aufl.). Wiesbaden: VS.

Wolter, J. (2017). Disziplinierungspraktiken in der Grundschule: Formalisierung sozialer Bezugnahme und Egalisierung von Differenz. In J. Budde \& N. Weuster (Hrsg.), Erziehung in Schule. Persönlichkeitsbildung als Dispositiv (S. 93-114). Wiesbaden: Vieweg.

Züchner, I., \& Fischer, N. (2014). Kompensatorische Wirkungen von Ganztagsschulen - Ist die Ganztagsschule ein Instrument zur Entkopplung des Zusammenhangs von sozialer Herkunft und Bildungserfolg? Zeitschrift für Erziehungswissenschaft, 17(2), 349-367. 